Abanico Agroforestal. Enero-Diciembre 2021; 3:1-17. http://dx.doi.org/10.37114/abaagrof/2021.9 Revisión de Literatura. Recibido: 11/05/2021. Aceptado: 27/11/2021. Publicado: 28/12/2021. Clave: e2021-35.

\title{
Reflexiones sobre la importancia del uso del inglés en la ciencia y en la educación
}

\author{
Reflections on the importance of English language use of in science and education
}

\section{Julio Carrillo-Beltrán ${ }^{1 \mathrm{ID}}$, Nadia Abad-Matos ${ }^{2}$ ID, Armando Ramírez-Jiménez ${ }^{3 \mathrm{ID}}$, Verónica Llamas-Rodríguez ${ }^{4 \mathrm{ID}}$, Carmen Llanos-Ramírez ${ }^{1 \mathrm{ID}}$, Sinahí Gómez- Campos $^{1 \mathrm{ID}}$, Socorro Salgado-Moreno ${ }^{*} \mathrm{ID}$}

\begin{abstract}
${ }^{1}$ Unidad Académica de Contaduría y Administración. Universidad Autónoma de Nayarit. Nayarit, México. ${ }^{2}$ Instituto Nacional de Ciencias Agrícolas. San José de las Lajas, Mayabeque, Cuba. ${ }^{3}$ Unidad Académica de Unidad Académica de Turismo y Gastronomía. Universidad Autónoma de Nayarit. Nayarit, México. ${ }^{4}$ Secretaría de Investigación y Posgrado. Universidad Autónoma de Nayarit. Nayarit, México. ${ }^{5}$ Unidad Académica de Medicina Veterinaria y Zootecnia. Universidad Autónoma de Nayarit. Nayarit, México. *Autor de correspondencia: Socorro Salgado-Moreno. Universidad Autónoma de Nayarit, Unidad Académica de Medicina Veterinaria y Zootecnia. Km 3.5 Carretera Compostela-Chapalilla. Compostela, Nayarit, México. CP. 63700. E-mail: doctorjuliocesarcarrillobeltran@uan.edu.mx, nadia770309@gmail.com, armando@uan.edu.mx, vllamas@uan.edu.mx, carmen.llanos@uan.edu.mx, sinahi.gomez@uan.edu.mx,coco_salgado@hotmail.com.
\end{abstract}

\section{Resumen}

En las últimas 2 décadas el idioma inglés ha cobrado cada día más relevancia en el mundo digital, académico, tecnológico, cultural, educativo y por supuesto en el campo de la investigación científica. La globalización incluye un idioma tan universal que se pueden comunicar un poco más de 7 mil millones de habitantes que estamos viviendo hoy en día en el planeta Tierra. Por consiguiente, el desarrollo de las naciones está estrechamente relacionado con las habilidades, competencias, conocimientos, saberes y estudios profesionales con los que cuentan sus ciudadanos. Una herramienta esencial es tener un elevado conocimiento de las 4 habilidades del idioma inglés de acuerdo al marco común europeo de referencia que es el estándar internacional que define una competencia lingüística que posee una persona con respecto a cualquier idioma. El objetivo del presente artículo es el enunciar de forma cualitativa haciendo una comparativa con distintas investigaciones sobre la importancia del uso del inglés en los distintos campos de la ciencia, analizando los métodos de enseñanza más efectivos de este idioma y su relevancia en la ahora llamada sociedad de la información, sociedad del conocimiento o sociedad postmoderna que ya se ha convertido una exigencia en el campo de la investigación científica.

Palabras clave: globalización, investigación, inglés, habilidades, ciencia.

\begin{abstract}
In the last 2 decades the English language has become more and more relevant in the digital, academic, technological, cultural, educational, and of course in the field of scientific research. Globalization includes a language so universal that a little more than 7 billion inhabitants living today on planet Earth can communicate. Therefore, the development of nations is closely related to the skills, competences, knowledge, and professional studies that their citizens have and an essential tool is to have a high knowledge of the 4 skills of the English language according to the Common European Framework of Reference which is the international standard that defines a linguistic competence that a person possesses with respect to any language. The aim of this article is to enunciate in a qualitative way making a comparative with different researches about the importance of the English use in the different fields of science, analyzing the most effective teaching methods of the English language and its relevance in the now called information society, knowledge society or postmodern society that has already become a requirement in the field of scientific research.
\end{abstract}

Keywords: globalization, research, English, skills, science. 


\section{INTRODUCCIÓN}

El presente artículo tiene como finalidad principal mostrar la importancia de conocer el idioma inglés que según la Organización de las Naciones Unidas (United Naciones, 2021) está considerado el cómo idioma oficial en el mundo. En un mundo globalizado donde resulta sumamente necesario comunicarse de forma rápida, masiva ante una sociedad tan insaciable de consumir información de forma constante y vertiginosa; por lo anterior, el idioma inglés se posiciona como lengua fundamental para adquirir y difundir conocimientos de cualquier índole. Este idioma es las publicaciones periódicas, en revistas electrónicas de alto impacto internacional, libros y conferencias académicas, es el lenguaje dominante más usado como segunda lengua y la lengua franca de comunicación científica. Por lo consiguiente, en la presente investigación se analiza la relación tan sobresaliente que el idioma inglés tiene con el desarrollo, la historia, la divulgación y la enseñanza de las ciencias, con énfasis en la investigación científica; así como la relevancia de este idioma en el quehacer de los científicos en dichas áreas y a su vez las oportunidades y retos que el idioma inglés conlleva en la enseñanza en todos los niveles educativos. Como bien es sabido este siglo XXI nos ha traído grandes avances en rubros tales como: la tecnología, la ciencia y la globalización lo que sin duda ha ido generando cambios en el entorno que impactan tanto de forma positiva como negativa, y los estudiantes de instituciones de educación superior durante toda su formación profesional deben de estar preparados para ser sumamente competitivos fundamentalmente ser conscientes de la relevancia del idioma inglés.

Si bien el español es el idioma nativo de más personas que el inglés, este último es más aprendido como segundo idioma que el primero. Estimaciones recientes sugieren que unos 402 millones de personas lo hablan como su primer idioma, así como es utilizado en la actualidad por más de 1000 millones de hablantes no nativos (Ortiz, 2013).

La globalización es sin lugar a dudas hoy en día uno de los principales factores que detonan el desarrollo económico, social, educativo en innovación tecnológica lo que hace que este idioma tenga relevancia en todas las naciones del mundo.

$\mathrm{Si}$ los profesionistas de cualquier programa académico de todas las instituciones superiores a nivel internacional tuvieran el conocimiento nivel intermedio de una segunda lengua estaremos ante una nueva sociedad competitiva, más desarrollada en todos los rubros mencionados anteriormente y sobre todo que tengan más oportunidades laborales.

La importancia de tener dominio del inglés radica en que es hablado a nivel internacional. A lo largo y ancho del globo terráqueo, en todas las naciones del mundo se le ha nombrado "El idioma universal", a pesar de que no es una lengua oficial en la mayoría de los países, actualmente "Es el idioma que más se está enseñando como segunda 
legua alrededor del mundo (Quezada, 2011). Es una realidad que en el contexto de la globalización actual que vivimos, la transferencia de conocimiento también es esencialmente plurilingüe (Edmondson, 2003), en este siglo XXI se orienta por su influencia tecnológica, científica, económica, sus tratados internacionales y en el mundo de las finanzas hacia el inglés.

Al respecto, en el área científica el inglés es preponderante: actualmente, dos terceras partes de los científicos del mundo leen en dicha lengua y la utilizan como un medio para dar a conocer sus investigaciones (Niño-Puello, 2013). Ante este escenario resulta evidente que el inglés es fundamental para adquirir conocimiento. Asimismo, en el área educativa, este idioma es primordial si se pretende capacitar a las nuevas generaciones para insertarse y competir adecuadamente en un mundo globalizado.

El inglés como lengua generadora de conocimiento científico; a pesar de que a nivel internacional la proporción de pobladores de habla inglesa pueda ser menor a la de otras lenguas (por ejemplo, chino o español), en el contexto globalizado actual el inglés tiene un dominio y difusión indiscutible. Este idioma tiene estatus oficial o especial en más de 75 países (Agudelo, 2011).

Así pues, con el contexto planteado en este orden de ideas, está claro que el idioma inglés es fundamental para adquirir conocimiento. Esto se debe a que es el lenguaje fundamental de libros, revistas electrónicas de alto impacto internacional, publicaciones periódicas y conferencias académicas, en ámbitos de ciencia y tecnología. Un aproximado del $65 \%$ de científicos en el mundo leen en inglés, y al menos tres cuartos de la información electrónica recabada y almacenada en bases de datos están en este idioma (Edmondson, 2003).

Es decir, que la mayoría de los países escogen este idioma para enseñar como lengua extranjera debido al alcance que tiene dentro de la comunicación y la investigación.

Por ello, las exigencias profesionales y personales que impone este mundo globalizado fuerzan a la universidad a ponerle especial atención a la formación de profesionales bilingües; por cuanto en la actualidad el manejo de un segundo idioma, como es el inglés, pasó de ser un valor agregado a constituirse en una competencia lingüística que requiere desarrollar el individuo (España, 2010).

De allí la obligación de las universidades por promover el aprendizaje del idioma inglés y lograr que forme parte del currículo educacional, haciendo que los alumnos desarrollen nuevas capacidades para que sean competentes ante la demanda que exige el mercado laboral de hoy en día. 
Es innegable que existe un vínculo muy estrecho entre el uso y la importancia del inglés en la investigación y en las publicaciones científicas. El uso de esta lengua internacional en el área de la ciencia está bien documentado. El inglés ha trascendido culturas y ha influenciado desde la moda hasta las tradiciones y estilos de vida (Crystal, 2004).

El idioma inglés por lo anteriormente expuesto en este artículo nos muestra claramente que en la investigación científica es fundamental comprender textos, artículos científicos, y obviamente también escribirlo. Seguramente más del $90 \%$ de producción de los resultados que se publican en la actualidad son en inglés cualquiera que sea el país en donde se haya realizado la investigación. Con certeza se puede afirmar que los artículos publicados en el idioma inglés tienen amplia difusión, citación y aceptación en la comunidad científica (Ammon, 2001). Por lo tanto, en la producción de investigaciones científicas se debiera contar con las competencias y habilidades suficientes tanto orales como escritas para alcanzar un nivel idóneo en un mayor dominio de las 4 habilidades propias de este idioma, aunque mayormente la comprensión lectora de textos científicos como la producción escrita y el uso adecuado de la gramática inglesa en un contexto especializado con una especial terminología, con el objetivo principal de incrementar los conocimientos y habilidades propias a esta actividad profesional como es la comunicación con otros especialistas en su rama de conocimiento, el envío de una ponencia a un evento nacional o internacional y la lectura o presentación de un artículo o trabajo escrito a una revista científica.

\section{DESARROLLO}

El grado más alto de la evolución lingüística lo constituye el lenguaje oral, y de todas las especies que existen en el planeta tierra el ser humano es el único que lo tiene desarrollado. Regularmente se utiliza como instrumento de comunicación, representación y de relación social y es de una importancia trascendental para el desarrollo cognitivo, social y afectivo del individuo, de aquí, el papel tan importante que desempeña el lenguaje oral y justamente llega a su cúspide en la definición de competencia de comunicación lingüística. De esta forma, la comunicación humana alcanza toda su originalidad cuando se ejerce a través del lenguaje, incluso si existen otros medios de comunicación, sobre todo hoy en este mundo que vivimos actualmente. El lenguaje es el único tipo de conducta social cuya función primaria es la comunicación (Verón, 1971). Por consiguiente, el lenguaje evoluciona y se convierte en una de las mayores riquezas para la interacción de las personas con su entorno, ya que es el principal medio de comunicación entre las mismas. El lenguaje es la primera manifestación específica del hombre como tal, es decir, como ente capaz de conocer el mundo, así como la primera forma, y la única absolutamente general de la que el hombre dispone para fijar y objetivar más acá de las impresiones y reacciones inmediatas, el conocimiento del mundo y de sí mismo, o sea, todo el contenido de la conciencia 
(Coseriu, 1967). En consecuencia, el registro evolutivo del hombre como ente social por medio del análisis de la historia del lenguaje se puede decir que el lenguaje es un proceso de comunicación que permite la emisión y recepción de información. Kristeva (1988), confirma que el lenguaje es un proceso de comunicación basado en la emisión de un mensaje entre por lo menos dos sujetos hablantes, siendo el uno el emisor, y el otro el receptor. El lenguaje es, por tanto, la capacidad propia de la especie humana para comunicarse por medio de un sistema de signos vocales, que pone en juego una técnica corporal compleja y supone la existencia de una función simbólica (Lewandowski, 2000). El lenguaje "es precisamente la elaboración y transmisión de información mediante la lengua lo que permite la interacción social, la comunicación" (Garrido, 1994). Por ende, todo lo que se produce en relación con el lenguaje sucede para ser comunicado en el intercambio social. De lo anterior expuesto se deduce que el código lingüístico es el instrumento que nos permite conocer el mundo que nos rodea e interpretarlo, para así poder desenvolvernos en él. Porque, según Malmberg (1966) nuestra manera de emplear sonidos y palabras, formas y construcciones sintácticas, es asimismo una parte del complejo de los modelos sociales de conducta que caracterizan a los grupos humanos.

Todo análisis y reflexión que se hace sobre la cultura y la vida social, bajo cualquiera de sus formas, es también un análisis del lenguaje humano, de las convenciones, del sistema lingüístico. Los modelos sociales y culturales se reflejan en la estructura de la lengua, es decir, en el código lingüístico que da origen al lenguaje (Coseriu, 1967).

Por todo lo mencionado anteriormente se observa la importancia que siempre le ha dado el ser humano a la comunicación que cabe señalar que ha sido un factor determinante en su evolución, ha sido inherente al ser humano y por supuesto que ha permitido al hombre tener acceso a la cultura y al mundo que lo rodea. Siguiendo a Flores \& Orozco (2005), se puede definir que la comunicación es una palabra que proviene de la voz latina "comunicare" que quiere decir, poner o puesto en común.

La comunicación es considerada la herramienta por medio de la cual el hombre puede modificar su entorno. También ha sido para el ser humano el vehículo de transmisión tanto de ideas, pensamientos, sentimientos y reflexiones sobre el pasado y de opiniones acerca de su porvenir o futuro.

El ser humano es por naturaleza un ser social y la comunicación es algo sumamente vital para él, ya no podría vivir sin la interacción con los demás, por su necesidad constante de expresar sentimientos, pensamientos, palabras, emociones a veces a través del uso de símbolos. El origen del lenguaje en el ser humano es milenario al igual que su deseo por comunicarse, está inherente también la necesidad de investigar, de explorar, de querer conocer y de aprender otras actividades, nuevas, lúdicas, que sean interesantes, que parezcan desafiantes, que vayan aderezadas con el dinamismo propio del tiempo 
generacional en el que se vive, un ejemplo claro de lo anteriormente expuesto es el aprendizaje de un nuevo idioma, como desafío, reto, meta u objetivo; siempre será para el ser humano una prueba de valentía, de voluntad y de atrevimiento. Es por ello que han surgido en los últimos dos siglos una diversidad de métodos para el aprendizaje de idiomas.

Con respecto al aprendizaje de lengua extranjera, Richards \& Rodgers (2001) entienden el concepto de enfoque de enseñanza como un conjunto de creencias y principios que se pueden utilizar como base para enseñar un idioma. Se caracterizan por la diversidad de interpretaciones en cuanto al modo en que se pueden aplicar y pueden ser revisados y actualizados en el transcurso del tiempo según aparezcan nuevas prácticas. Es un diseño o sistema docente específico basado en una teoría concreta de la lengua y del aprendizaje de idiomas.

El Marco común europeo de referencia establece una escala de seis niveles comunes de referencia para la organización del aprendizaje de lenguas y homologación de los distintos títulos emitidos por las entidades certificadas. La división se agrupa en tres bloques que responden a una división más clásica de nivel básico, intermedio y avanzado, aunque no se corresponden exactamente con los niveles clásicos por estar situados por encima o por debajo de ellos, ver Tabla 1 (Instituto Cervantes, 2001).

Para una mejor compresión y socialización utilizando el idioma inglés debemos favorecer en nuestros programas educacionales un elevado proceso enseñanza-aprendizaje donde los estudiantes universitarios logren al menos ubicarse a partir del tercer nivel de referencia para su mejor desempeño como profesional posteriormente.

Según la Doctora en Ciencias Hernández Chérrez (2014), el inglés es sin duda la lengua que más se enseña en el mundo, se lo enseña como lengua extranjera en más de 100 países como: China, Rusia, Alemania, España, Egipto, Brasil y Ecuador. En la Unión Europea el inglés es una lengua obligatoria en 14 países o regiones empezando en la etapa de primaria. En el 2009, el $73 \%$ del alumnado matriculado en primaria en la Unión Europea estaba aprendiendo el inglés. En la educación secundaria obligatoria el porcentaje superó el $90 \%$ y en la formación preprofesional y profesional de grado medio alcanzó un $74.9 \%$.

En la educación superior se espera que los estudiantes recién graduados sean capaces de comprender textos complejos que se encuentren dentro de su área de conocimiento y puedan expresarse con cierto grado de fluidez cuando se comuniquen con otras personas en inglés, por tal razón las Instituciones de Educación Superior deben garantizar el cumplimiento de un número de horas básicas y la calidad en las cátedras que son impartidas (Ministerio de Educación Nacional de Colombia, 2005). 
Tabla 1. Escala de seis niveles comunes de referencia para la organización del aprendizaje de lenguas extranjeras

\begin{tabular}{|c|c|c|}
\hline Nivel & Subnivel & Descripción \\
\hline \multirow[t]{2}{*}{$\begin{array}{c}\text { A } \\
\text { (Usuario } \\
\text { básico) }\end{array}$} & $\begin{array}{c}\mathbf{A 1} \\
\text { (Acceso) }\end{array}$ & $\begin{array}{l}\text { Es capaz de comprender y utilizar expresiones cotidianas de uso } \\
\text { muy frecuente, así como frases sencillas destinadas a satisfacer } \\
\text { necesidades de tipo inmediato. Puede presentarse a sí mismo y a } \\
\text { otros, pedir y dar información personal básica sobre su domicilio, } \\
\text { sus pertenencias y las personas que conoce. Puede relacionarse de } \\
\text { forma elemental siempre que su interlocutor hable despacio y con } \\
\text { claridad y esté dispuesto a cooperar. }\end{array}$ \\
\hline & $\begin{array}{c}\text { A2 } \\
\text { (Plataforma) }\end{array}$ & $\begin{array}{l}\text { Es capaz de comprender frases y expresiones de uso frecuente } \\
\text { relacionadas con áreas de experiencia que le son especialmente } \\
\text { relevantes (información básica sobre sí mismo y su familia, } \\
\text { compras, lugares de interés, ocupaciones, etc). Sabe comunicarse } \\
\text { a la hora de llevar a cabo tareas simples y cotidianas que no } \\
\text { requieran más que intercambios sencillos y directos de información } \\
\text { sobre cuestiones que le son conocidas o habituales. Sabe describir } \\
\text { en términos sencillos aspectos de su pasado y su entorno, así como } \\
\text { cuestiones relacionadas con sus necesidades inmediatas. }\end{array}$ \\
\hline \multirow[t]{2}{*}{$\begin{array}{c}\text { B } \\
\text { (Usuario } \\
\text { independiente) }\end{array}$} & $\begin{array}{c}\text { B1 } \\
\text { (Intermedio) }\end{array}$ & $\begin{array}{l}\text { Es capaz de comprender los puntos principales de textos claros y } \\
\text { en lengua estándar si tratan sobre cuestiones que le son conocidas, } \\
\text { ya sea en situaciones de trabajo, de estudio o de ocio. Sabe } \\
\text { desenvolverse en la mayor parte de las situaciones que pueden } \\
\text { surgir durante un viaje por zonas donde se utiliza la lengua. Es } \\
\text { capaz de producir textos sencillos y coherentes sobre temas que le } \\
\text { son familiares o en los que tiene un interés personal. Puede describir } \\
\text { experiencias, acontecimientos, deseos y aspiraciones, así como } \\
\text { justificar brevemente sus opiniones o explicar sus planes. }\end{array}$ \\
\hline & $\begin{array}{l}\text { B2 (Intermedio } \\
\text { alto }\end{array}$ & $\begin{array}{l}\text { Es capaz de entender las ideas principales de textos complejos que } \\
\text { traten de temas tanto concretos como abstractos, incluso si son de } \\
\text { carácter técnico siempre que estén dentro de su campo de } \\
\text { especialización. Puede relacionarse con hablantes nativos con un } \\
\text { grado suficiente de fluidez y naturalidad de modo que la } \\
\text { comunicación se realice sin esfuerzo por parte de ninguno de los } \\
\text { interlocutores. Puede producir textos claros y detallados sobre } \\
\text { temas diversos, así como defender un punto de vista sobre temas } \\
\text { generales indicando los pros y los contras de las distintas opciones. }\end{array}$ \\
\hline \multirow[t]{2}{*}{$\begin{array}{c}\text { C } \\
\text { (Usuario } \\
\text { competente) }\end{array}$} & $\begin{array}{l}\text { C1 (Dominio } \\
\text { operativo } \\
\text { eficaz) }\end{array}$ & $\begin{array}{l}\text { Es capaz de comprender una amplia variedad de textos extensos y } \\
\text { con cierto nivel de exigencia, así como reconocer en ellos sentidos } \\
\text { implícitos. Sabe expresarse de forma fluida y espontánea sin } \\
\text { muestras muy evidentes de esfuerzo para encontrar la expresión } \\
\text { adecuada. Puede hacer un uso flexible y efectivo del idioma para } \\
\text { fines sociales, académicos y profesionales. Puede producir textos } \\
\text { claros, bien estructurados y detallados sobre temas de cierta } \\
\text { complejidad, mostrando un uso correcto de los mecanismos de } \\
\text { organización, articulación y cohesión del texto. }\end{array}$ \\
\hline & C2 (Maestría) & $\begin{array}{l}\text { Es capaz de comprender con facilidad prácticamente todo lo que } \\
\text { oye o lee. Sabe reconstruir la información y los argumentos } \\
\text { procedentes de diversas fuentes, ya sean en lengua hablada o } \\
\text { escrita, y presentarlos de manera coherente y resumida. Puede } \\
\text { expresarse espontáneamente, con gran fluidez y con un grado de } \\
\text { precisión que le permite diferenciar pequeños matices de significado } \\
\text { incluso en situaciones de mayor complejidad. }\end{array}$ \\
\hline
\end{tabular}


La mayoría de la bibliografía que utilizan los estudiantes fundamentalmente los universitarios son presentados en idioma inglés principalmente aquellas que están más actualizadas por lo que al no saber este idioma les falta la herramienta necesaria para investigar y la preparación del estudiante no sería completa ni integral.

Aunque los principios básicos de la redacción científica se aplican en todas las lenguas, es muy común que su escritura se realice en inglés con vistas a contribuir a sus mayores visibilidad y alcance posibles, ya que este se ha constituido la lengua internacional de la ciencia, situación por tanto que precisa un mayor cuidado en su preparación, sobre todo, en el caso de los que no son angloparlantes para garantizar la debida calidad del manuscrito correspondiente y por ende, la mayor posibilidad de aceptación para su publicación por revistas de reconocido prestigio internacional. Resulta aconsejable escribir de forma sencilla, evitar las construcciones complejas y los textos recargados para garantizar una comunicación eficaz (Núñez, 2009).

Cuando se efectúan búsquedas bibliográficas para diseñar nuevos proyectos de investigación (más aún, cuando se trata de temas novedosos y poco investigados), la cantidad de información que se encuentra en esta lengua supera de forma significativa la información disponible en otros idiomas, incluyendo el español. De igual forma, muchas revistas internacionales indexadas con los mejores rankings exigen que los artículos científicos que corresponden a los resultados de los proyectos de investigación sean publicados en inglés (Uribe, 2012).

El concepto de métodos de enseñanza en lenguas extranjeras ha tenido hasta el momento diversas interpretaciones y definiciones. Richards \& Rodgers (2001) sugieren la siguiente definición: El método es un plan general para la presentación ordenada de material lingüístico, ninguna de las cuales contradice, y cual se basa en el enfoque seleccionado. Un enfoque es axiomático, un método es procedimental. Dentro de un enfoque, puede haber muchos métodos.

Los autores Richards JC \& Rodgers TS. (2001), Harmer J (2011), Diane Larsen-Freeman (2000), Mehisto P, Marsh D. \& Frigols MJ. (2008), Echevarria J, Vogt ME \& Short DJ. (2008) han hecho una recopilación a lo largo de muchos años, definen y mencionan los métodos de enseñanza del idioma inglés que han sido durante décadas los más efectivos a nivel internacional cuando han sido aprendidos por personas que tienen como lengua materna una distinta obviamente que el idioma inglés:

- El Método de Gramática y Traducción. Este método de enseñanza funcionó como la norma hasta la década de 1960. Fue utilizado con el propósito de ayudar a los estudiantes a leer y apreciar la literatura de lenguas extranjeras. También se esperaba que, a través del estudio de la gramática de la lengua meta, los estudiantes se familiarizaran más con la gramática de su lengua materna y que esta familiaridad les 
ayudara a hablar y escribir mejor su lengua materna.

- Método directo o natural. El Método directo se hizo muy popular cuando el método de traducción gramatical no se consideró eficaz para aprender a utilizar una lengua extranjera con fines comunicativos. Este método tiene una regla muy básica: no permite la traducción. De hecho, el Método directo recibe su nombre por el hecho de que el significado debe transmitirse directamente en el idioma meta mediante el uso de ayudas visuales y de demostración, sin recurrir a la lengua materna de los estudiantes.

- Método Audio lingual. Una de las razones para el desarrollo del Método Audio-lingual fue la entrada de los Estados Unidos en la Segunda Guerra Mundial. Además, a medida que los Estados Unidos emergieron como potencia internacional, hubo una demanda creciente de enseñanza de inglés a inmigrantes y estudiantes extranjeros. En el método audio-lingual se puso mayor énfasis en el dominio de los aspectos formales del lenguaje, es decir, un buen uso de la gramática. Bajo esta metodología la gramática o las estructuras gramaticales son el punto de partida de la enseñanza, principalmente a través de la producción oral intensiva con atención en la pronunciación. La Metodología audio-lingual tiene sus bases en las teorías conductistas de Skinner sobre el aprendizaje (Estímulo-Respuesta-Modelo de reforzamiento).

- Enfoque Comunicativo. En la década de 1980 surge el enfoque comunicativo (CLT). Sus orígenes son el resultado de la insatisfacción de educadores y lingüistas con el Método de Gramática y Traducción y el Método Audio-lingual. El enfoque comunicativo hace de la competencia comunicativa el objetivo de la enseñanza de las lenguas y reconoce la interdependencia del lenguaje y la comunicación. Enfatiza la importancia de las funciones del lenguaje en lugar de centrarse únicamente en la gramática y el vocabulario. Un principio rector de este enfoque es enseñar a los estudiantes el uso correcto de las formas del lenguaje para que puedan aplicarse en una variedad de contextos y para una variedad de propósitos. CLT es considerado como un enfoque y no un método ya que es compatible con muchos métodos de enseñanza. No existe una metodología de enseñanza específica disponible para CLT. La disponibilidad de una metodología de enseñanza especifica sería propio de un método. La teoría subyacente del lenguaje de CLT considera al lenguaje como comunicación, y el objetivo de la enseñanza es el desarrollo de la competencia comunicativa.

- El Aprendizaje del Lenguaje en Comunidad (Community Language Learning). A principios de los años 70 Charles, A. Curran propuso el aprendizaje del lenguaje en comunidad como un método cuyo principio básico es establecer relaciones interpersonales entre el docente y los alumnos, para facilitar el aprendizaje. La base de esta metodología es la idea de que el aprendizaje es aprender a vivir, es un asunto social y puede producirse solo en la interacción social. Esta metodología se basa en técnicas 
de asesoramiento para el aprendizaje. El rol de los docentes se centra en disminuir los temores e inseguridades, así como atender los problemas personales y lingüísticos que la persona encuentra en el aprendizaje de lenguas extranjeras.

- El Método Respuesta Física Total (Total Physical Response). La Respuesta Física Total se basa en la idea de James J. Asher, quien plantea que cuanto más activo es el aprendizaje, más eficaz se vuelve. Con base en sus estudios de investigación, (Asher,1969) Según este autor la manera más rápida es manifestar la comprensión y menos estresante de lograr el entendimiento de cualquier idioma como lengua extranjera es seguir instrucciones pronunciadas por quien ejerza la función docente (sin traducción del idioma nativo). Asimismo, el propulsor de TPR, trabajó desde la premisa de que el aprendizaje de una lengua extranjera por parte de adultos podría tener patrones de desarrollo similares a los de la adquisición del lenguaje infantil. Si los niños aprenden su lenguaje a partir de un discurso dirigido a ellos en forma de órdenes e instrucciones para realizar acciones, entonces los adultos también aprenderán mejor de esa manera. En consecuencia, TPR propone a los alumnos responder físicamente a la lengua que escuchan. El procesamiento del lenguaje se combina con la acción física.

- Instrucción basada en contenido (Content Based Instruction). Content Based Instruction (CBI) fue desarrollado en la década de 1980 y se basa en los principios del enfoque comunicativo: es un enfoque de enseñanza en el cual se organiza la enseñanza en función de contenidos o temas a ser enseñados y no de un tipo de programa lingüístico, gramatical establecido. Como eje principal, la enseñanza se basa en el contenido, y su objetivo primario es transmitir contenidos significativos a los estudiantes. El lenguaje se utiliza para enseñar el tema, y el lenguaje mismo se adquiere como un subproducto del aprendizaje sobre el contenido del mundo real.

- Enseñanza de lenguas basadas en tareas (Task Based Learning). El enfoque basado en tareas de la enseñanza de idiomas fue introducido en la década de 1980 y es un desarrollo del enfoque comunicativo. Fue desarrollado por Dave y Jane Willis y se basa en el uso de las tareas como la unidad central de la planificación y la enseñanza de lenguas. El objetivo primario es proporcionar a los alumnos un contexto natural para el uso del lenguaje. A medida que trabajan para completar una tarea, tienen oportunidades de interactuar. Tal interacción se piensa para facilitar la adquisición del lenguaje. Al realizar la tarea comprueban si han comprendido correctamente y, a veces, tienen que pedir aclaraciones. Al interactuar y socializar con otros, llegan a escuchar un lenguaje que puede estar más allá de su capacidad en ese momento, pero que puede ser asimilado en su conocimiento de la lengua meta para su uso en un momento posterior. En lugar de una estructura lingüística, se les presenta a los alumnos una tarea que tienen que realizar o un problema que tienen que resolver. 
- El modelo PPP (Presentation-Practice-Production). El modelo PPP (acrónimo de presentación, práctica y producción), surgió a partir de la enseñanza estructuralsituacional. En este procedimiento el docente introduce una situación que contextualiza el lenguaje a enseñar. El modelo PPP se implementa a través de la progresión de tres secuencias: 1) Presentación: el docente presenta palabras o estructuras nuevas, da ejemplos, los escribe en el pizarrón, etc. 2) Práctica: los alumnos practican el uso de palabras o estructuras de manera controlada, por ejemplo, haciendo que las oraciones formen mensajes, preguntando y respondiendo preguntas, dando oraciones basadas en una imagen. La práctica puede ser oral o escrita. 3) Producción: los alumnos usan el lenguaje que han aprendido para expresarse más libremente, hablando o escribiendo sobre sus propias vidas e intereses, expresando opiniones, o imaginándose en diferentes situaciones. Al igual que la práctica, la producción puede ser oral o escrita.

- El modelo SIOP (Sheltered Instruction Observation Protocol Model). Echevarria J, Vogt ME \& Short DJ, (2008) crearon el SIOP (Sheltered Instruction Observation Protocol o Protocolo de Observación del Inglés Protegido, se utiliza en contextos en los que hay alumnos que tienen que aprender contenidos en una lengua que no es su lengua materna Al combinar la enseñanza basada en el contenido y el SIOP, los docentes buscan desarrollar el dominio del idioma inglés de los alumnos mediante la incorporación de información de las áreas temáticas que probablemente estudiarán. La enseñanza protegida, que forma parte de la abreviatura SIOP, puede explicarse como un conjunto de estrategias de enseñanza diseñadas para docentes que enseñan contenido académico a alumnos con diferentes niveles de habilidades lingüísticas. Además de la información comprensible, SIOP ofrece diferentes estrategias de aprendizaje y técnicas de andamiaje. Las estrategias y técnicas de aprendizaje sirven de ayuda para que los docentes puedan elegir diferentes métodos de enseñanza acordes al objetivo de una clase y promover el pensamiento crítico y estratégico de los alumnos.

- El aprendizaje integrado de contenidos e idiomas (CLIL-Content and Language Integrated Learning). Content and Language Integrated Learning (CLIL) es una metodología de enseñanza de los idiomas cuyo énfasis principal no está en la forma, sino en el contenido. El acrónimo CLIL fue presentado por Marsh (2002), que trabaja en el área del multilingüismo y de la educación bilingüe en la Universidad Finlandesa de Jyväskylä, y define esta metodología como una pedagogía del lenguaje centrada en el significado que contrasta con las que se centran en la forma. Así pues, este mismo autor sugirió que CLIL fuera un término genérico para referirse a metodologías que conducen a la educación enfocada en el aprendizaje y en los contenidos.

- El método audiovisual es un modelo didáctico concebido para la enseñanza de la LE ha principiantes. Da prioridad al lenguaje oral, sin por ello desatender el lenguaje escrito; las primeras sesiones (aproximadamente 20 horas) del método se dedican al lenguaje 
oral y a continuación se comienza el aprendizaje del lenguaje escrito. Se considera que el aprendizaje de la LE se canaliza a través del oído (escuchando diálogos) y de la vista (observando la situación); ello explica el empleo combinado de grabaciones de diálogos en soporte magnético (en la época inicial, en magnetófono; posteriormente, en casete) e imágenes en filminas (parecidas a las diapositivas). Los recursos materiales reciben una atención especial, se procura que la presentación resulte lo más atractiva posible. A. P. Howatt (1987) define el método como «el primer intento serio de construir una descripción pedagógica de una lengua extranjera, basándose en transcripciones de conversaciones habladas. Durante el presente artículo se evidencia la importancia de la globalización de la que todos formamos parte como ciudadanos que somos de cualquier nación, pero también, como parte de una sociedad que tiene amplias posibilidades y es prácticamente imposible no darse cuenta de lo que acontece alrededor, ya que existe una gran variedad de formas de comunicación masiva disponibles al alcance de un click en los dispositivos electrónicos. Lo mismo sucede con el idioma inglés, al cual desde edad temprana un ser humano tiene contacto directa o indirectamente. Se ha venido analizando que en todos los niveles educativos forma parte del plan de estudios de las escuelas tanto públicas como privadas, ya sea, de forma intensiva o con pocas horas al mes de clases de inglés, pero, invariablemente siempre habrá un contacto ligero pero permanente. Se ha mencionado también que el ser humano siempre utiliza el lenguaje para comunicarse por distintas formas muy creativas hoy en día en esta era digital. Después, cuando culmina su carrera universitaria, es profesionista y le apasiona la investigación científica de nueva cuenta va a necesitar tener el conocimiento del idioma inglés como segunda lengua. En el presente artículo se han analizado diferentes puntos de vista de autores expertos en este tema y también al final del desarrollo del mismo se han mencionado todos los métodos de enseñanza más utilizados, más populares y más efectivos para la adquisición y dominio del idioma inglés. La ciencia es un excelente puente que seguirá contribuyendo en el desarrollo económico de la sociedad para recuperarnos lo más pronto posible de esta experiencia que todos hemos vivido como ha sido la pandemia Covid-19,también brinda la oportunidad de acercarnos al conocimiento, al saber, el fomentar hábitos de disciplina en el estudio científico; a través de los trabajos científicos contribuir de alguna manera en la formación de ciudadanos más reflexivos, analíticos, empáticos y que cada uno de ellos a través de valores éticos, responsabilidad, honestidad, creatividad e ingenio salgamos adelante con la premisa de que la educación, la ciencia y la tecnología son el camino adecuado que nos permita estar preparados como sociedad para futuras contingencias de cualquier índole procurando siempre el bienestar de todos y cada uno de los ciudadanos. 


\section{METODOLOGÍA}

La presente revisión acerca de la importancia del uso del inglés en la ciencia se ha desarrollado principalmente con un enfoque cualitativo, tanto de tipo narrativo como descriptivo generando una nueva perspectiva desde un punto de vista subjetivo, analizando mediante razonamiento lógico una serie de comparativas que llevan a un discernimiento de lo más relevante e importante de conceptos y definiciones del tópico en cuestión. A su vez, la investigación es de tipo exploratorio también, ya que durante el desarrollo se ha hecho la revisión sistemática de la literatura de diversos artículos científicos tanto en inglés como en español sobre este tema mediante el empleo de las bases de datos científicas: Direct Science, Emerald, Scopus, Scielo, Redalyc, Google Académic. Así mismo, para el presente artículo se aplicó criterios de búsqueda en base a los motores o descriptores, alcanzando los mejores resultados con artículos de alto impacto. La matriz de la presente investigación se desarrolla con el propósito de correlacionar cada uno de los elementos mediante la ejecución de una secuencia lógica (Rivero, 2013).

\section{CONCLUSIONES}

El presente artículo confirma que la mayor parte de quienes publican a nivel internacional trabajos o artículos científicos se ven beneficiados en cierta forma por la citación en revistas de alto impacto. Cabe destacar que, la labor intelectual de investigación científica que se realizan en instituciones de educación superior a través de la publicación de trabajos científicos en inglés representa por sí mismo un gran esfuerzo, especialmente por parte de quienes cuya lengua materna es distinta al idioma inglés o quienes se encuentran en países no anglosajones, es un claro ejemplo de la globalización que está sucediendo en el mundo actual al formar un nuevo conocimiento. La gran ventaja en este siglo XXI es que está plagado de una gran tecnología que está a la vanguardia, pero, aún no se ha podido reemplazar de ninguna manera la labor del docente quien enseña inglés como figura que orienta o facilita el aprendizaje a través de estrategias didácticas efectivas, interactivas, visuales y con recursos didácticos que aporten al aprendizaje significativo con ingenio y creatividad, los docentes siguen siendo entonces una parte fundamental, por supuesto que los recursos digitales y tecnológicos que se siguen utilizando ayudan a quienes desean aprender mediante el método autodidacta, lo facilita la tecnología con la que contamos hoy en día, aunado a esto están los gadgets y múltiples dispositivos electrónicos que conectados a internet facilitan el aprendizaje con aplicaciones gratuitas o videos en YouTube.

Los investigadores científicos seguirán enfrentándose a un gran desafío, tiempos de cambio siempre son favorables, para seguir aprendiendo y esto sin lugar a dudas renueva el quehacer propio de quien o quienes investigan en un área muy apasionante como lo es la ciencia, se dice por ahí renovarse o morir y todos ahora debemos buscar 
y explorar las mejores técnicas o métodos de aprendizaje del idioma más popular, importante y útil en el último siglo para seguir contribuyendo con los trabajos científicos en el desarrollo económico, educativo, tecnológico, cultural, académico y aportando siempre con innovación en un mundo donde todo está en constante movimiento y la clave es estar en constante aprendizaje, adaptación y capacitación especialmente en las tecnologías de comunicación.

\section{LITERATURA CITADA}

AGUDELO JH. 2011. Publicar en inglés. Revista Colombiana de Ciencias Pecuarias. 24(11).

http://aprendeenlinea.udea.edu.co/revistas/index.php/rccp/article/view/324624/2078190 0

AMMON U. 2001. Editor's preface, in U. Ammon (ed.), The dominance of English as a language of science: Effects on other languages and language communities. Pp. 5-10. Berlin: Mouton de Gruyter. ISBN:13-978-3110166477.

ASHER JJ. 1969. The Total Physical Response Approach to Second Language Learning. The Modern Language Journal. 53(1):3-17. https://doi.org/10.2307/322091

INSTITUTO Cervantes. 2001. Centro Virtual Cervantes. Niveles comunes de referencia: escala global.

https://cvc.cervantes.es/ensenanza/biblioteca_ele/marco/cap_03_01.htm

COSERIU E. 1967. L'arbitraire du signe. Zur Spätgeschicte eines aristotelischen Begriffes. In Archiv für das Studium der neueren Sprachen und Literaturen. 204:81-112. http://www.romling.uni-tuebingen.de/coseriu/publi/coseriu39.pdf

CRYSTAL D. 2004. The Stories of English. Londres, Reino Unido: Allen Lane. ISBN: 07139-9752-4.

DIANE LARSEN-FREEMAN. 2000. Techniques and Principles in Language Teaching. Oxford, England: Oxford University Press. Second Edition. Pp. 189. https://doi.org/10.18172/jes.83

ECHEVARRIA J, Vogt ME, Short DJ. 2008. Making content comprehensible for English learners: The SIOP Model. New York, USA: Pearson.

https://doi.org/10.1080/15235882.1999.10162746 
EDMONDSON AC. 2003. Framing for Learning: Lessons in Successful Technology Implementation. California Management Review. 45(2):34-54.

https://doi.org/10.2307/41166164

HERNÁNDEZ E. 2014. El B-learning como estrategia metodológica para mejorar el proceso de enseñanza-aprendizaje de los estudiantes de inglés de la modalidad semipresencial del departamento especializado de idiomas de la Universidad Técnica de Ambato. Tesis Doctoral. Departamento de Didáctica y Organización Escolar, Facultad de Educación, Universidad Complutense de Madrid. España. Pp. 503. https://eprints.ucm.es/id/eprint/29610/1/T35913.pdf

ESPAÑA C. 2010. El idioma inglés en el currículo universitario: importancia, retos y alcances. Revista Electrónica Educare. XIV (2):63-69. https://www.redalyc.org/articulo.oa?id=194115606005

FLORES de Gortari S, Orozco Gutiérrez E. 2005. Hacia una comunicación administrativa e integral. México: Ed Trillas. ISBN: 9682440564.

GARRIDO Medina J. 1994. Idioma e información. La lengua española de la comunicación. Madrid, España: Síntesis. ISBN: 84-7738-222-0.

https://dialnet.unirioja.es/servlet/libro?codigo=226549

HARMER J. 2011. The Practice of English Language Teaching. England: Longman. ISBN:13-9780582046566.

HOWATT APR. 1987. From structural to communicative. En Kaplan, R. B (ed.) (1988). Annual Review of Applied Linguistics, 8. Cambridge: C. U. P.

https://cvc.cervantes.es/ensenanza/biblioteca_ele/diccio_ele/diccionario/metodoaudiovi sual.htm

KRISTEVA Julia. 1988. El lenguaje, ese desconocido: Introducción a la lingüística. Madrid, España: Fundamentos.

https://introduccionlenguaje2010.files.wordpress.com/2010/09/kristeva-julia-el-lenguajeese-desconocido.pdf

LEWANDOWSKI T. 2000. Diccionario de lingüística. Madrid, España: Cátedra. ISBN: 978-84-376-0363-6 84-376-0363-3. 
MALMBERG B. 1966a. Les nouvelles tendances de la linguistique (versi6n francaise de 'New Trends') Paris. Francia. https://www.persee.fr/doc/bude_00045527_1969_num_1_1_3044_t1_0139_0000_2

MARSH D. 2002. Content and Language Integrated Learning: The European DimensionActions, Trends and Foresight Potential. Retrieved from:

http://europa.eu.int/comm/education/languages/index.html

MEHISTO P, Marsh D, Frigols MJ. 2008. Uncovering CLIL: Content and Language Integrated Learning in Bilingual and Multilingual Education. Oxford: Macmillan URI. http://hdl.handle.net/11162/64524

MINISTERIO de Educación Nacional de Colombia. 2005. Bilingüismo: estrategia competitividad. http://www.mineducacion.gov.co/1621/article-97497.html

NIÑO-PUELLO M. 2013. El inglés y su importancia en la investigación científica: algunas reflexiones. Revista Colombiana de Ciencia Animal. 5(1):243-254.

https://doi.org/10.24188/recia.v5.n1.2013.487

NÚÑEZ Jesús A. 2009. Algunas reglas para escribir un buen artículo científico en inglés. Revista CENIC, Ciencias biológicas. 4(1): 33-34.

https://revista.cnic.cu/index.php/RevBiol/article/view/656

ORTIZ I. 2013. La importancia del idioma inglés en la educación. El Nuevo Diario. Managua, Nicaragua. http://www.elnuevodiario.com.ni/desde-la-u/305910-importanciaidiomaingles-educacion/

QUEZADA R. 2011. Biografía de la lengua inglesa: Cómo nació, a qué se dedica, y... ¿Pensando en retirarse? Revista Destiempos. 32:3-17. http://www.destiempos.com/n32/rquezada.pdf

RICHARDS JC, Rodgers TS. 2001. Approaches and Methods in Language Teaching. Second edition. Cambridge, England: Cambridge University Press. Online ISBN 9780511667305. https://doi.org/10.1017/CBO9780511667305

RIVERO Cárdenas I, Gómez Zermeño M, Abrego Tijerina F. 2013. Tecnologías educativas y estrategias didácticas: criterios de selección. Educación y Tecnología. 3:190-206. http://revistas.umce.cl/index.php/edytec/article/view/134

UNITED NATIONS. 2021. Secretary-General's Guidance on Behavioral Science. https://www.un.org/en/content/behaviouralscience/ 
URIBE JD. 2012. Importancia del idioma inglés en las instituciones de educación superior: el caso de la Corporación Universitaria de Sabaneta. Uni-pluriversidad. 12(2):97-103. ISSN: 1657-4249.

https://dialnet.unirioja.es/servlet/articulo?codigo $=7580368$

VERÓN E. 1971. Introducción: Hacia una ciencia de la comunicación social. En Lenguaje y Comunicación social. Buenos Aires, Argentina: Nueva Visión. https://www.redalyc.org/journal/3239/323961444002/323961444002.pdf 